Article

\title{
Immunogenicity of eGFP-Marked Recombinant Lactobacillus casei against Transmissible Gastroenteritis Virus and Porcine Epidemic Diarrhea Virus
}

\author{
Meiling Yu ${ }^{1,+}$, Li Wang ${ }^{1,+}$, Sunting Ma ${ }^{1}$, Xiaona Wang ${ }^{1}$, Yusai Wang ${ }^{1}$, Ya Xiao ${ }^{1}$, \\ Yanping Jiang ${ }^{1}$, Xinyuan Qiao ${ }^{1,2}$, Lijie Tang ${ }^{1,2}$, Yigang $\mathrm{Xu}{ }^{1,2, *}$ and Yijing $\mathrm{Li}^{1,2, *}$ \\ 1 College of Veterinary Medicine, Northeast Agricultural University, Harbin 150030, China; \\ yu19890130@126.com (M.Y.); wanglicau@163.com (L.W.); masunting@163.com (S.M.); \\ xiaonawang0319@163.com (X.W.); bggfgg163@163.com (Y.W.); 18845756200@163.com (Y.Xi.); \\ jiangyanping2017@126.com (Y.J.); qiaoxinyuan@126.com (X.Q.); tanglijie@neau.edu.cn (L.T.) \\ 2 Heilongjiang Key Laboratory for Animal Disease Control and Pharmaceutical Development, \\ Harbin 150030, China \\ * Correspondence: yigangxu@neau.edu.cn (Y.Xu.); yijingli@163.com (Y.L.); Tel./Fax: +86-451-5519-0363 (Y.L.) \\ + These authors contributed equally to this work.
}

Received: 3 August 2017; Accepted: 23 September 2017; Published: 25 September 2017

\begin{abstract}
Porcine transmissible gastroenteritis virus (TGEV) and porcine epidemic diarrhea virus (PEDV) are the causative agents of highly fatal acute diarrhea in pigs, resulting in enormous losses in the pig industry worldwide. To develop an effective bivalent oral vaccine against TGEV and PEDV infection, the D antigenic site of the TGEV spike (S) protein and the major antigen site (core neutralizing epitope-COE) of the PEDV S protein were used as immunogens, and the enhanced green fluorescent protein (eGFP) gene was used as a reporter to construct genetically engineered Lactobacillus casei LLPGG $^{\mathrm{F}}$-T7g10-eGFP-6D-COE. The expression of proteins of interest by the recombinant $L$. casei was confirmed by confocal laser scanning microscopy and a Western blot assay, and the immunogenicity of $\mathrm{rLpPG}^{\mathrm{F}}-\mathrm{T} 7 \mathrm{~g} 10-\mathrm{eGFP}-6 \mathrm{D}-\mathrm{COE}$ in orally immunized mice was evaluated. The results showed that levels of anti-PEDV and anti-TGEV serum immunoglobulin G (IgG) and mucosal secreted immunoglobulin A (sIgA) antibodies obtained from the mice immunized with $\mathrm{rLpPG}^{\mathrm{F}}$-T7g10-eGFP-6D-COE, as well as the proliferation levels of lymphocytes, were significantly higher than those in mice orally administered phosphate-buffered saline (PBS) or rLpPG-T7g10. Moreover, the serum IgG antibodies showed neutralizing effects against PEDV and TGEV. Our data suggest that the antibiotic resistance-free genetically engineered $L$. casei bivalent oral vaccine provides a safe and promising strategy for vaccine development against PEDV and TGEV.
\end{abstract}

Keywords: transmissible gastroenteritis virus; porcine epidemic diarrhea virus; Lactobacillus casei; antibiotic-free selection; immunogenicity

\section{Introduction}

Porcine transmissible gastroenteritis virus (TGEV) and porcine epidemic diarrhea virus (PEDV), which belong to the genus Coronavirus, family Coronaviridae, and order Nidovirales, are the main pathogens that cause highly fatal acute diarrhea in newborn piglets. This is followed by vomiting, dehydration, and high mortality, resulting in enormous losses in the pig industry worldwide [1]. Currently, porcine transmissible gastroenteritis (TGE), first reported in the United States in 1933, has spread to numerous European, South American, and Asian countries [2-4]. Porcine epidemic diarrhea (PED), which has been sporadic or locally endemic over the past three decades, experienced a 
larger-scale outbreak in the United States in 2013. Herds vaccinated with the CV777-inactivated vaccine were also infected, resulting in tremendous losses to the swine industry [5-7]. Accumulating evidence indicates that this large-scale recurrence of PED was caused by highly virulent PEDV variants [8-11]. In addition, co-infection of TGEV and PEDV often causes higher morbidity and mortality in newborn piglets. Therefore, the development of a safe and highly efficient vaccine against TGE and PED would be of great importance.

It is now clear that the gastrointestinal mucosas are the primary sites of PEDV and TGEV infection $[12,13]$ and that mucosal immunization is a promising way to prevent PEDV and TGEV infection. However, the existing live attenuated/inactivated vaccines administered parenterally cannot effectively induce mucosal immunity against PEDV and TGEV infection [14-17]. Although some vaccines for TGE and PED developed in China can effectively stimulate intestinal mucosal immunity through Houhai acupoint injection, this immunization procedure is more time-consuming and labor-intensive. Therefore, vaccines that can induce a mucosal immune response against PEDV and TGEV would be of great significance, in particular those demonstrated to be safe, inexpensive, easy to use, and effective. The spike (S) glycoproteins of TGEV and PEDV possess antigen epitopes, and previous reports have demonstrated that the D antigen site (amino acids 378-392) in the S protein of TGEV and the core neutralizing epitope (COE; amino acids 499-638) in the S protein of PEDV can elicit neutralizing antibodies against TGEV and PEDV infection, respectively. This suggests that these are promising candidate antigens for the development of a genetically engineered vaccine $[12,18,19]$.

Lactobacillus casei (L. casei) is a potential delivery vehicle for oral vaccines, because it is a probiotic bacterium characterized by its safety and resistance to gastric acid and bile $[20,21]$. Previous reports have shown that recombinant $L$. casei live vaccine is able to colonize the murine intestines for five days [22] and the swine intestines for longer [23]. Moreover, we have previously constructed a recombinant $L$. casei live vaccine expressing the $D$ antigen site of the TGEV S glycoprotein combined with muramyl dipeptide and tuftsin as adjuvants, suggesting the possibility of a promising oral vaccine against TGEV challenge [12]. However, plasmid-mediated antibiotic resistance is commonly used as a selective marker for genetically engineered bacteria [24-26]. This could result in potential biosafety issues due to the transfer of antibiotic resistance from genetically engineered bacteria to environmental pathogens. Enhanced green fluorescent protein (eGFP) is a luminescent jellyfish protein with the amino acid substitutions necessary to generate a strong fluorescence signal when excited by ultraviolet or blue light. It is widely used in biological research, including in studies of cell differentiation, gene tracking, and protein localization and operation in vivo $[27,28]$, providing a potential candidate for a screening marker to replace antibiotic resistance.

In this study, a genetically engineered L. casei strain, rLpPGF-T7g10-eGFP-6D-COE, was constructed using the TGEV S protein D antigen site and PEDV S protein-neutralizing antigen epitope region COE as immunogens, L. casei 393 as an antigen delivery vehicle, and eGFP as a selective marker, combined with a constitutive expression plasmid, pPG-T7g10. The immunogenicity of this strain when orally administered in mice was evaluated, suggesting a potential approach for the prevention of TGEV and PEDV infection.

\section{Materials and Methods}

All applicable international and national guidelines for the care and use of animals were followed. Approval (2016NEFU-315, 13 April 2017) was obtained from the Institutional Committee of Northeast Agricultural University for the animal experiments.

\subsection{Virus, Plasmid, Bacterium, and Cell Lines}

TGEV strain TH98 and PEDV strain HLJ-2012 were isolated by our laboratory from PEDV/TGEV-positive samples collected from pig farms in which a severe outbreak of acute diarrhea had been reported in piglets $[29,30]$. L. casei ATCC 393 was kindly provided by Jos Seegers (NIZO Institute, Netherlands). African green monkey kidney cells (Vero cells; ATCC CCL-81) and swine 
testicle (ST) cells were purchased from the China Center for Culture Collection (Wuhan, China) and were cultured in Dulbecco's modified Eagle medium (DMEM; Gibco, Gaithersburg, MD, USA) supplemented with $10 \%$ fetal bovine serum (FBS; Gibco) at $37{ }^{\circ} \mathrm{C}$ with $5 \% \mathrm{CO}_{2}$. The details of all plasmids used in this study are listed in Table 1.

Table 1. Details of plasmids used in this study.

\begin{tabular}{|c|c|c|}
\hline Plasmids & Relevant Characteristics & Description/Reference \\
\hline pMD18-T-6D & As a template for PCR to amplify DNA fragment $6 D$ & Liu et al. 2011 [31] \\
\hline peGFP-N1 & As a template for PCR to amplify DNA fragment $e G F P$ & Wang et al. 2016 [32] \\
\hline pMD18-T-COE & $\begin{array}{l}3169 \mathrm{bp} ; A m p^{r} \text {; core neutralizing epitope (COE) of PEDV strain } \\
\text { HLJ-2012 }\end{array}$ & This study \\
\hline pMD19-Ts & $2692 \mathrm{bp} ; A m p^{r}$ & Takara (DaLian, China) \\
\hline pMD19-Ts-6D & $\begin{array}{l}3325 \mathrm{bp} ; A m p^{r} ; \text { the amplified DNA fragment } 6 D \text { was inserted } \\
\text { into pMD19-Ts }\end{array}$ & This study \\
\hline pMD19-Ts-eGFP & $\begin{array}{l}3469 \mathrm{bp} ; A m p^{r} ; \text { the amplified DNA fragment } e G F P \text { was } \\
\text { inserted into pMD19-Ts }\end{array}$ & This study \\
\hline pMD18-T-6D-COE & $3766 \mathrm{bp} ; A m p^{r} ; 6 D$ was inserted into pMD18-T-COE & This study \\
\hline pPG-T7g10 & $\begin{array}{l}4965 \mathrm{bp} ; \mathrm{Cm}^{r} ; \text { HCE promoter of Geobacillus toebii D-AAT; } \\
\text { poly- } \gamma \text {-glutamate synthase A (PgsA) anchor; constitutive } \\
\text { expression plasmid }\end{array}$ & Song et al., 2014 [33] \\
\hline pPG-T7g10-6D-COE & $6034 \mathrm{bp} ; \mathrm{Cm}^{r} ; 6 \mathrm{D}-\mathrm{COE}$ was inserted into pPG-T7g10 & This study \\
\hline pPG-T7g10-eGFP-6D-COE & $6793 \mathrm{bp} ; \mathrm{Cm}^{r} ; e G F P$ was inserted into pPG-T7g10-6D-COE & This study \\
\hline pPGF$^{\mathrm{F}}$-T7g10-eGFP-6D-COE & $6596 \mathrm{bp} ; \mathrm{Cm}^{r}$ gene of pPG-T7g10-eGFP-6D-COE was deleted & This study \\
\hline
\end{tabular}

\subsection{Construction of the eGFP-Marked Recombinant Lactobacillus Strain}

The primers used for amplifying genes encoding 6D (a peptide of the the $\mathrm{D}$ antigenic site of the TGEV spike (S) protein was repeated six times), COE, and eGFP are listed in Table 2. The linker sequence (GGGGS)3 was added in primers Fs2 and Re. A schematic diagram of the DNA plasmid construction is shown in Figure 1. In brief, the gene encoding 6D was inserted into plasmid pMD18T-COE at SacI and MluI sites, generating plasmid pMD18T-6D-COE; then, the fusion DNA fragment 6D-COE, obtained from pMD18T-6D-COE by SacI and ApaI digestion, was inserted into the corresponding sites of plasmid pPG-T7g10, generating recombinant plasmid pPG-T7g10-6D-COE. Next, the gene encoding eGFP was inserted into pPG-T7g10-6D-COE at SacI and KpnI sites, generating recombinant plasmid pPG-T7g10-eGFP-6D-COE; finally, a chloramphenicol resistance $\left(\mathrm{Cm}^{r}\right)$ gene selective marker in pPG-T7g10-eGFP-6D-COE was deleted using restriction enzymes $S t u \mathrm{I}$ and $\mathrm{NcoI}$, followed by blunt-end treatment and ligation, giving rise to recombinant plasmid $\mathrm{pPG}^{\mathrm{F}}$-T7g10-eGFP-6D-COE. All recombinant plasmids were identified by restriction enzyme digestion and sequencing. 
For construction of the recombinant Lactobacillus strain, L. casei 393 competent cells were prepared according to a method previously described [34], followed by electroporation. Briefly, $50 \mathrm{ng}$ of recombinant plasmid pPG $^{\mathrm{F}}$-T7g10-eGFP-6D-COE was gently mixed with $200 \mu \mathrm{L}$ of L. casei 393 competent cells at $4{ }^{\circ} \mathrm{C}$ for $1 \mathrm{~min}$; then, the mixture was transferred into a pre-cooled Gene Pulser (Bio-Rad, Hercules, CA, USA) disposable cuvette (inter-electrode distance of $0.2 \mathrm{~cm}$ ) and subjected to a single electric pulse $(1.5 \mathrm{~V} ; 200 \Omega ; 25 \mu \mathrm{F})$ with a Gene Pulser (Bio-Rad). After growth at $37^{\circ} \mathrm{C}$ for $8 \mathrm{~h}$, the recombinant Lactobacillus strain with green fluorescence signal was collected through flow cytometry using a FACSCalibur (BD Biosciences, San Diego, CA, USA) at $488 \mathrm{~nm}$ and was grown on an de Man-Rogosa-Sharpe (MRS) plate at $37^{\circ} \mathrm{C}$ for $36 \mathrm{~h}$. This was followed by PCR confirmation and a chloramphenicol sensitivity assay, giving rise to recombinant strain $\mathrm{LLPFG}^{\mathrm{F}}$-T7g10-eGFP-6D-COE. The hereditary stability of recombinant $L$. casei strains was detected, and rLpPG $^{\mathrm{F}}$-T7g10-eGFP-6D-COE was analyzed for stability by serially transferring the cultures after $24 \mathrm{~h}$ of incubation into MRS medium at $37^{\circ} \mathrm{C}$ ( $1 \%$ inoculum; 50 generations). Plasmids were extracted from the cells, and PCR was used to confirm the presence of the gene eGFP-6D-COE in the plasmid using the primers Fe and Rs2.

Table 2. Details of primers used in this study.

\begin{tabular}{|c|c|c|c|c|}
\hline Gene & ID & Primer Sequences & Length(bp) & $\begin{array}{l}\text { Accession } \\
\text { No./Reference }\end{array}$ \\
\hline \multirow{2}{*}{$6 \mathrm{D}$} & Fs1 & 5'-GAGCTCGCAGGTACCAGATCTTGTT-3' & \multirow{2}{*}{612} & \multirow{2}{*}{ Liu et al., 2011 [31] } \\
\hline & Rs1 & 5'-ACGCGTGAGTCTAGAGGATCCGCCAC-3' & & \\
\hline \multirow{3}{*}{$\mathrm{COE}$} & & 5'-ACGCGTGGTGGAGGAGGTTCAGGCGGAGGTGGC & \multirow{3}{*}{420} & \multirow{3}{*}{ JX512907 } \\
\hline & Fs2 & TCTGGCGGTGGCGGATCGGTTACTTTGCCATCATTT-3' & & \\
\hline & Rs2 & 5'-GGGCCCAACGTCCGTGACACCTTC-3' $^{\prime}$ & & \\
\hline \multirow{2}{*}{ eGFP } & $\mathrm{Fe}$ & 5'-CGAGCTCATGGTGAGCAAGGGCGA-3' & \multirow{2}{*}{720} & \multirow{2}{*}{ U55762.1 } \\
\hline & $\operatorname{Re}$ & $\begin{array}{l}\text { 5'-GGGGTACCCGATCCGCCACCGCCAGAGCCACCTCC } \\
\text { GCCTGAACCGCCTCCACCCTTGTACAGCTCGTCCATGC-3' }\end{array}$ & & \\
\hline
\end{tabular}

Restriction enzyme recognition sites used for cloning are shown with underline. The linker (GGGGS)3 bases are shown in bold. 6D: a peptide of the $\mathrm{D}$ antigenic site of the porcine transmissible gastroenteritis virus spike (S) protein was repeated six times, COE: core neutralizing epitope in the $\mathrm{S}$ protein of porcine epidemic diarrhea virus, eGFP: enhanced green fluorescent protein. 

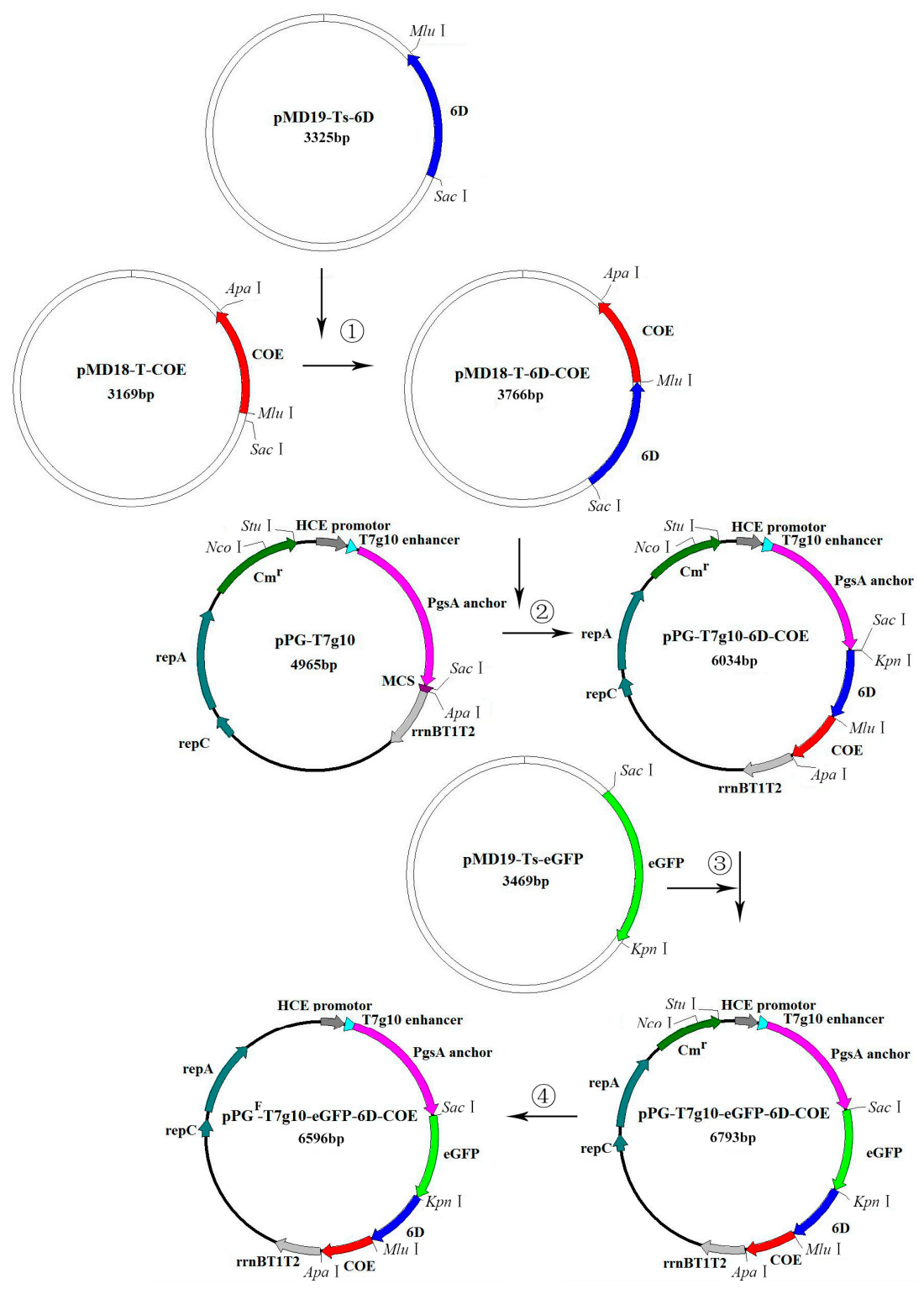

Figure 1. Schematic drawing of the construction of DNA plasmids. Plasmids pPG-T7g10-6D-COE and $\mathrm{pPG}^{\mathrm{F}}$-T7g10-eGFP-6D-COE were generated according to the steps indicated by the arrows. (1) The gene encoding 6D was inserted into plasmid pMD18-T-COE at SacI and MluI sites, generating plasmid pMD18-T-6D-COE; (2) fusion DNA fragment 6D-COE, obtained from pMD18-T-6D-COE by SacI and $A p a \mathrm{I}$ digestion, was inserted into the corresponding sites of plasmid pPG-T7g10, generating recombinant plasmid pPG-T7g10-6D-COE; (3) the gene encoding enhanced green fluorescent protein (eGFP) was inserted into pPG-T7g10-6D-COE at SacI and KpnI sites, generating recombinant plasmid pPG-T7g10-eGFP-6D-COE; (4) the chloramphenicol resistance $\left(\mathrm{Cm}^{r}\right)$ gene as a selective marker in pPG-T7g10-eGFP-6D-COE was deleted using restriction enzymes StuI and NcoI, followed by blunt-end treatment and ligation, giving rise to recombinant plasmid $\mathrm{pPG}^{\mathrm{F}}$-T7g10-eGFP-6D-COE.

\subsection{Identification of Proteins Expressed by the Recombinant Strain}

For analysis of the expression of proteins of interest by recombinant strains rLpPG-T7g10-6D-COE and $\mathrm{rLpPG}^{\mathrm{F}}$-T7g10-eGFP-6D-COE, the bacterial strains were grown in basal MRS broth at $37^{\circ} \mathrm{C}$ for $16 \mathrm{~h}$ (optical density of sample measured at a wavelength of $\left.600 \mathrm{~nm}\left(\mathrm{OD}_{600}\right) \approx 3.0\right)$ without shaking and were harvested by centrifugation at $10,000 \times g$ for $2 \mathrm{~min}$. Cells were washed twice with sterile phosphate-buffered saline (PBS; pH 7.4) and lysed using a Mini-BeadBeater (BioSpec, 
Bartlesville, OK, USA). After centrifugation, the same quantity of total protein in the supernatants of each sample was isolated by sodium dodecyl sulfate $12 \%$ polyacrylamide gel electrophoresis (SDS-PAGE) and was transferred onto polyvinylidene fluoride membranes, followed by development with mouse anti-6D monoclonal antibody/rabbit anti-COE polyclonal antibody (diluted at 1:500) prepared in our lab, or mouse anti-eGFP monoclonal antibody (ZSGB-BIO, Beijing, China; diluted at 1:4000). Horseradish peroxidase (HRP)-conjugated goat anti-mouse/rabbit IgG antibody (Sigma, St. Louis, MO, USA) was utilized as a secondary antibody, diluted at 1:5000. Immunoblots were visualized with chemiluminescent substrate reagent (Pierce, Rockford, IL, USA) according to the manufacturer's instructions.

Laser confocal microscopy was used to confirm the expression of fusion protein eGFP-6D-COE on the surface of rLpPG ${ }^{\mathrm{F}}$-T7g10-eGFP-6D-COE using rLpPG-T7g10-6D-COE as a control. Briefly, recombinant strains were cultured in MRS medium at $37^{\circ} \mathrm{C}$ for $16 \mathrm{~h}$; then $1 \mathrm{~mL}$ of culture was collected by centrifugation at $5000 \times g$ for $5 \mathrm{~min}$. The pellets were washed three times with PBS, re-suspended in $1 \mathrm{~mL}$ of PBS, and smeared on a microscope slide. Images were viewed by laser confocal microscopy (Zeiss, Oberkochen, Germany).

\subsection{Immunizations}

In order to evaluate the immunogenicity of recombinant strain $\mathrm{rLPPG}^{\mathrm{F}}-\mathrm{T} 7 \mathrm{~g} 10-\mathrm{eGFP}-6 \mathrm{D}-\mathrm{COE}$ used as an oral vaccine, 5-week-old female specific pathogen-free (SPF) BALB/c mice (derived from Mus Musculus) ( $n=60)$ were obtained from Liaoning Changsheng Biotechnology Co., Ltd. (Liaoning, China) and kept under SPF conditions for one week with free access to a standard chow diet and water, in accordance with institutional guidelines. Prior to oral administration, the recombinant Lactobacillus strains were cultured for $16 \mathrm{~h}$ in MRS medium without shaking, washed with sterile PBS, and re-suspended in PBS at a concentration of $10^{10} \mathrm{CFU} \mathrm{mL} \mathrm{mL}^{-1}$. SPF BALB/c mice were randomly divided into four groups (15 mice per group): PBS, rLpPG-T7g10, rLpPG-T7g10-6D-COE, and rLpPGF-T7g10-eGFP-6D-COE. The immunization dosages are shown in Table 3. The mice were immunized once a day for 3 consecutive days and boosted twice at 2 week intervals. After immunization, serum samples were collected from the immunized mice on days $0,7,14,21,28$ and 35, and were stored at $-20{ }^{\circ} \mathrm{C}$ until they were required for use. Mucosal lavage samples were obtained from the vaginas of the mice by washing with $200 \mu \mathrm{L}$ of sterile PBS (pH 7.4) and were stored at $-20^{\circ} \mathrm{C}$ until analysis. In addition, fecal samples were collected and treated according to a method previously described [35]. Briefly, a $0.1 \mathrm{~g}$ fecal pellet was suspended in $400 \mu \mathrm{L}$ of PBS containing $1 \mathrm{mmoL} \mathrm{L}^{-1}$ phenylmethylsulfonyl fluoride (Sigma) and $1 \%$ bovine serum albumin (BSA) and was then incubated at $4{ }^{\circ} \mathrm{C}$ for $16 \mathrm{~h}$. After centrifugation, the supernatants were stored at $-20^{\circ} \mathrm{C}$ until use.

Table 3. Groups and immunization dosages.

\begin{tabular}{cccc}
\hline Group & No. of Mice & Dose/Treatment & No. of Treatments \\
\hline Phosphate-buffered saline (PBS) & 15 & $200 \mu \mathrm{L}$ of PBS & 3 \\
rLpPG-T7g10 & 15 & $200 \mu \mathrm{L}$ of $10^{10} \mathrm{CFU} \mathrm{mL}^{-1}$ & 3 \\
rLpPG-T7g10-6D-COE & 15 & $200 \mu \mathrm{L}$ of $10^{10} \mathrm{CFU} \mathrm{mL}^{-1}$ & 3 \\
rLpPGF-T7g10-eGFP-6D-COE & 15 & $200 \mu \mathrm{L}$ of $10^{10} \mathrm{CFU} \mathrm{mL}^{-1}$ & 3 \\
\hline
\end{tabular}

$\mathrm{CFU} \mathrm{mL} \mathrm{m}^{-1}$ : colony-forming unit per $\mathrm{mL}$ of recombinant bacteria.

\subsection{ELISA Assay}

To detect TGEV- and PEDV-specific antibodies in the collected samples, polystyrene microtiter plates were coated with purified TGEV/PEDV for $12 \mathrm{~h}$ at $4{ }^{\circ} \mathrm{C}$, using cultured ST/Vero cells as a negative antigen control. After blocking with $5 \%$ skim milk at $37^{\circ} \mathrm{C}$ for $2 \mathrm{~h}$ and washing three times with PBS-0.1\% Tween 20 (PBST), serum and mucus samples serially diluted in PBS-1\% BSA were added to wells in triplicate, and then plates were incubated for $1 \mathrm{~h}$ at $37^{\circ} \mathrm{C}$. After washing with PBST, HRP-conjugated goat anti-mouse IgG or IgA antibody (Invitrogen, Carlsbad, CA, USA) was added to 
each well (1:5000) and incubated for an additional $1 \mathrm{~h}$ at $37^{\circ} \mathrm{C}$. The substrate o-phenylenediamine dihydrochloride (Sigma) was used for color development, and the absorbance was measured at $490 \mathrm{~nm}$.

\subsection{TGEV and PEDV Neutralization by Mouse Immune Sera}

The neutralizing capacities of serum antibodies obtained from the mice immunized with rLpPG ${ }^{\mathrm{F}}$-T7g10-eGFP-6D-COE were determined. Briefly, the 50\% tissue culture infective dose (TCID 50 ) values of TGEV and PEDV were detected by the Reed-Muench method. Serum antibodies collected from the vaccinated mice on day 35 post-immunization were diluted at 1:10-1:320 (in a total of $50 \mu \mathrm{L}$ ), mixed with an equal volume of PEDV or TGEV (100 TCID 50 per $100 \mu \mathrm{L})$ and incubated at $37^{\circ} \mathrm{C}$ for $1 \mathrm{~h}$. Then, the treated viruses were added to a confluent monolayer of Vero and ST cells cultured in 24-well plates. The cells were overlaid with $1 \%$ methylcellulose, and the plates were incubated at $37{ }^{\circ} \mathrm{C}$ in a $5 \% \mathrm{CO}_{2}$ atmosphere and examined daily for five days for TGEV- and PEDV-specific cytopathic effects (CPE).

\subsection{Antigen-Induced Proliferation of Lymphocytes In Vitro}

On day 35 post-immunization, splenocytes from three mice from each group were prepared for a lymphocyte proliferation assay as previously described [34]. Briefly, $100 \mu \mathrm{L}$ of splenocytes $\left(5 \times 10^{7}\right.$ cells $\left.\mathrm{mL}^{-1}\right)$ was suspended in Roswell Park Memorial Institute (RPMI) 1640 medium containing $10 \%$ fetal calf serum and then transferred to a 96-well flat-bottom plate; the cells were re-stimulated for $72 \mathrm{~h}$ with 1.0, 5.0 or $25 \mu \mathrm{g} \mathrm{mL}{ }^{-1}$ TGEV-6D protein and PEDV-COE protein (produced by Escherichia coli prepared in our lab), using $5.0 \mu \mathrm{g} \mathrm{mL}^{-1}$ concanavalin A (ConA) and culture medium as positive and negative controls, respectively. The plates were supplemented with $10 \mu \mathrm{L}$ of 3-(4,5-dimethylthylthiazol-2-yl)-2,5-diphenyltetrazoliumbromide (MTT) per well and incubated for an additional $4 \mathrm{~h}$, and then proliferation was measured using $\mathrm{OD}_{490}$ values. The experiment was carried out in triplicate, and the lymphocyte proliferation index in the spleen (SI) was calculated as the mean reading of triplicate antigen stimulation wells divided by the mean reading of triplicate negative control wells.

\subsection{Statistical Analysis}

Data are shown as the means \pm standard errors of three replicates per test in a single experiment repeated three times. Tukey's multiple comparison tests were used to analyze differences among the groups. A $p$-value of $<0.05$ was considered statistically significant, and $p<0.01$ was considered highly significant.

\section{Results}

\subsection{Construction of eGFP-Marked Recombinant Lactobacillus Strain}

Following flow cytometry screening and growth on antibiotic-free MRS plates, eGFP-marked recombinant Lactobacillus strain $\mathrm{rLpPG}^{\mathrm{F}}$-T7g10-eGFP-6D-COE was obtained and identified with colony PCR (Figure 2a). As shown in Figure 2b, the strain rLpPG ${ }^{\mathrm{F}}-\mathrm{T7g} 10-\mathrm{eGFP}-6 \mathrm{D}-\mathrm{COE}$ could not grow on MRS agar medium in the presence of chloramphenicol. The result of the hereditary stability of recombinant L. casei strains showed that rLpPG ${ }^{\mathrm{F}}-\mathrm{T7g} 10-\mathrm{eGFP}-6 \mathrm{D}-\mathrm{COE}$ is stable for more than 50 generations. 
a

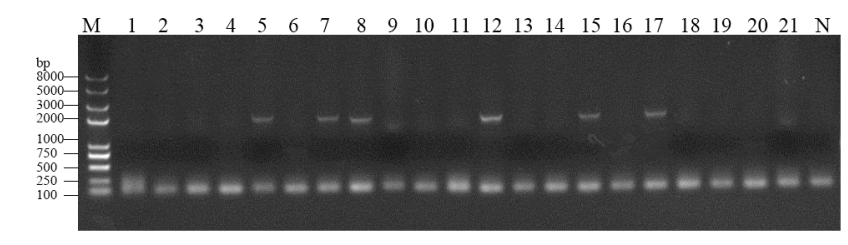

b
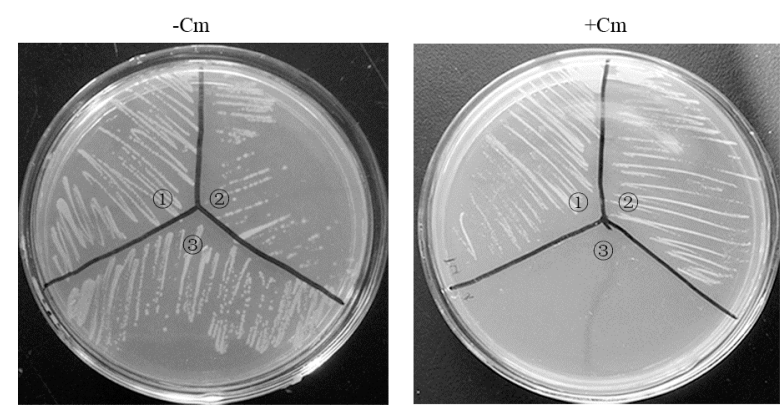

Figure 2. The identification of recombinant Lactobacillus strain rLpPGF-T7g10-eGFP-6D-COE without antibiotic resistance. (a) Recombinant strains with green fluorescence signal were collected, spread on an de Man-Rogosa-Sharpe (MRS) plate, and confirmed by colony PCR (Fe and Rs2 as primers). Lane M: Trans2K plus II DNA marker; lanes 1-21: colonies; lane N: negative control; (b) the results of chloramphenicol sensitivity assay. (1) rLpPG-T7g10-6D-COE; (2) rLpPG-T7g10-6D-COE; (3) $\mathrm{rLpPG}^{\mathrm{F}}-\mathrm{T} 7 \mathrm{~g} 10-\mathrm{eGFP}-6 \mathrm{D}-\mathrm{COE}$.

\subsection{Expression of Proteins of Interest by Recombinant Lactobacillus Strain}

For confirming the expression of the proteins of interest by the recombinant Lactobacillus strain constructed in this study, the cell lysates of recombinant strains rLpPG-T7g10-6D-COE and rLpPGF-T7g10-eGFP-6D-COE were analyzed by a Western blot assay. As shown in Figure 3, the expected immunoblot bands of fusion protein pgsA-6D-COE of $80 \mathrm{kDa}$ expressed by rLpPG-T7g10-6D-COE (Figure 3a,b) and the fusion protein pgsA-eGFP-6D-COE of $110 \mathrm{kDa}$ expressed by $\mathrm{rLpPG}^{\mathrm{F}}$-T7g10-eGFP-6D-COE were observed (Figure 3a-c), but these proteins were not expressed in rLpPG-T7g10 or L. casei. Moreover, green fluorescence could be observed by laser confocal microscopy on the surface of rLpPG ${ }^{\mathrm{F}}$-T7g10-eGFP-6D-COE but not on rLpPG-T7g10-6D-COE (Figure 4), indicating that eGFP could successfully be used as a selective marker.

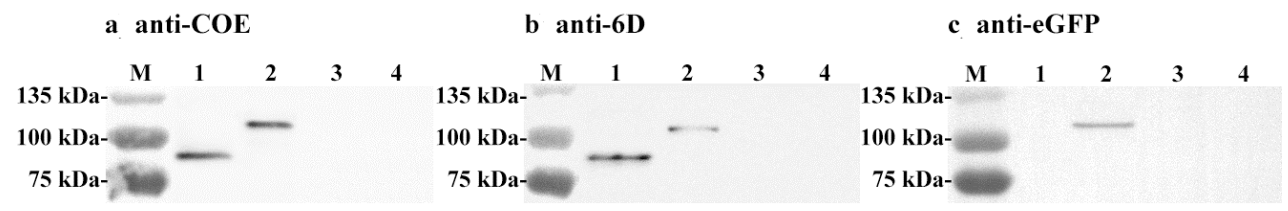

Figure 3. Western blot of recombinant Lactobacillus strain. Expression of poly- $\gamma$-glutamate synthase A (PgsA) fusion proteins by recombinant Lactobacillus was detected by Western blot with anti-COE (core neutralizing epitope) rabbit sera (a), anti-6D mouse sera (b), and anti-eGFP (enhanced green fluorescent protein) mouse antibodies (c). Lane 1: rLpPG-T7g10-6D-COE; lane 2: rLpPGF-T7g10-eGFP-6D-COE; lane 3: rLpPG-T7g10; lane 4: L. casei. 


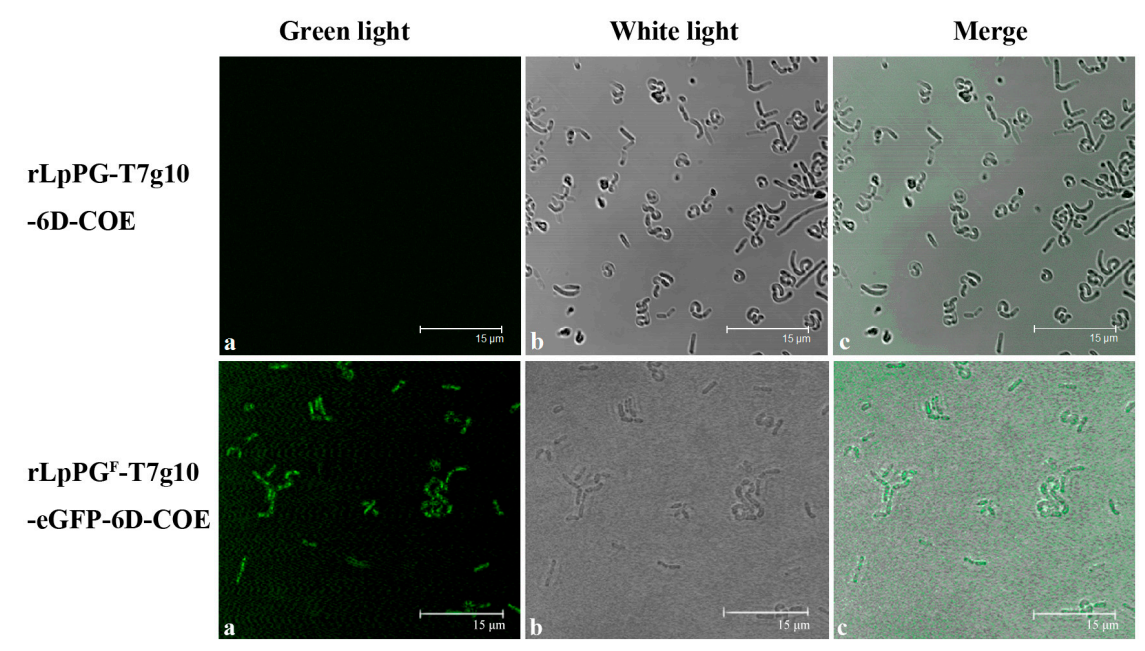

Figure 4. Expression of the PgsA-eGFP-6D-COE fusion protein on the cell surface. The localization of

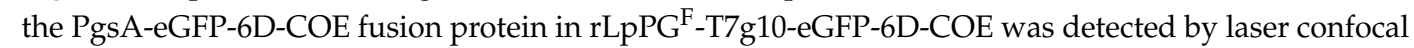
microscopy. Bars are $15 \mu \mathrm{m}$ for rLpPG ${ }^{\mathrm{F}}$-T7g10-eGFP-6D-COE and rLpPG-T7g10-6D-COE. Recombinant strains were observed by green light (a) and white light (b) respectively. Panel (c) is a merged image of panel $(\mathbf{a}, \mathbf{b})$.

\subsection{Immune Responses in Mice Induced by Recombinant Lactobacillus Strain}

Anti-TGEV/PEDV-specific secreted IgA (sIgA) and IgG antibodies were assessed to evaluate the ability of rLpPG-T7g10-6D-COE and rLpPGF-T7g10-eGFP-6D-COE to induce mucosal and systemic immune responses using BALB/c mice as a model. As shown in Figure 5, anti-TGEV/PEDV-specific antibodies were detected at high levels 7 days post-immunization and were significantly increased after the booster immunization, peaking at 35 days post-immunization. The levels of anti-TGEV/PEDV-specific mucosal sIgA in mouse feces and vaginas, as well as the levels of anti-TGEV/PEDV-specific serum IgG antibody in mice orally immunized with rLpPG-T7g10-6D-COE/rLpPG ${ }^{\mathrm{F}}$-T7g10-eGFP-6D-COE, were significantly higher $(p<0.01)$ than those in the PBS or rLpPG-T7g10 groups. In addition, the levels of anti-TGEV/PEDV sIgA in the vaginas of mice orally immunized with $\mathrm{rLpPG}$-T7g10-eGFP-6D-COE 35 days post-immunization were significantly higher $(p<0.01)$ than those in the rLpPG-T7g10-6D-COE group. There was no statistical difference $(p>0.05)$ observed in the PBS or rLpPG-T7g10 groups before and after immunization. 

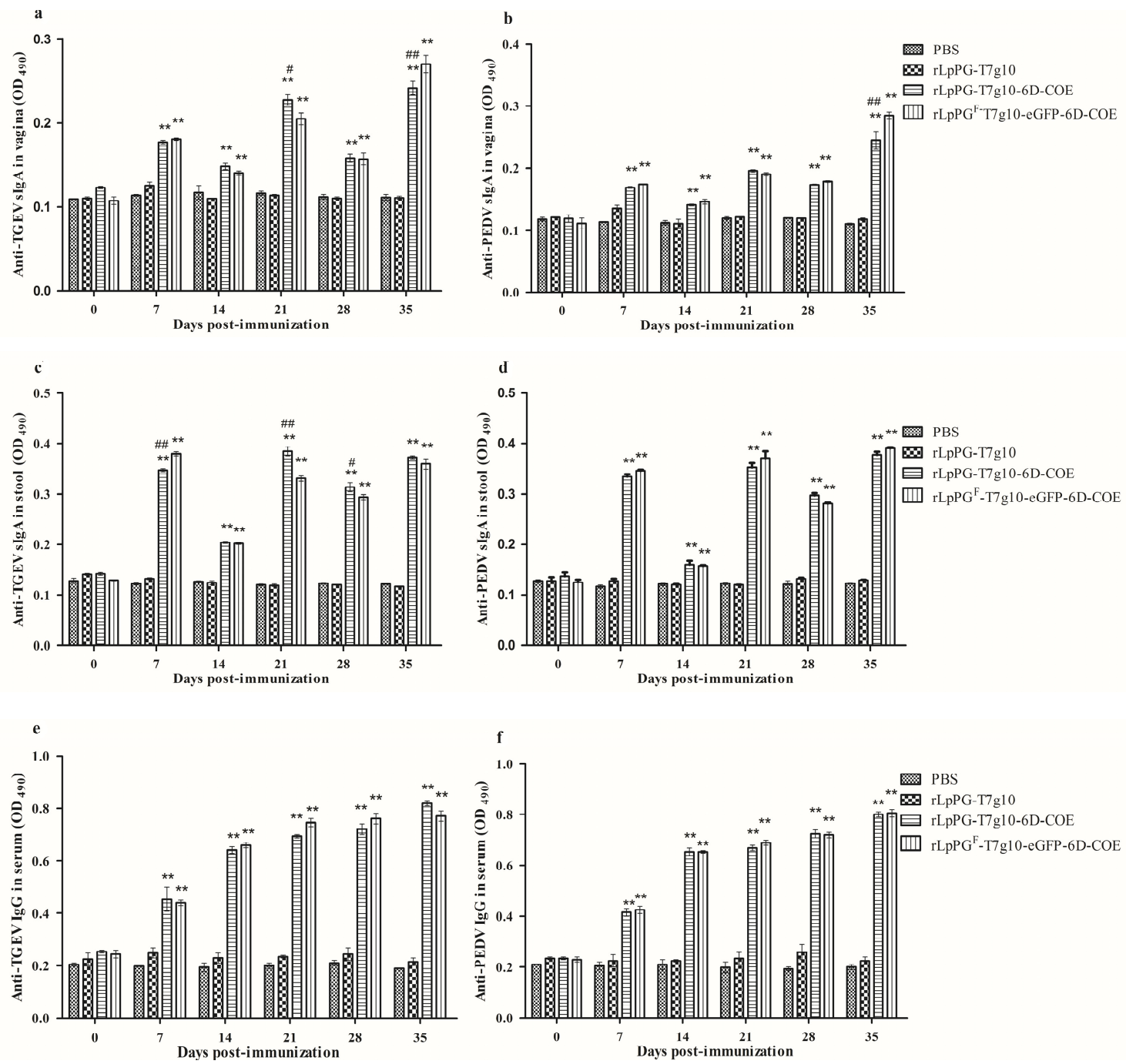

Figure 5. Levels of secreted immunoglobulin A (sIgA) and immunoglobulin G ( $\operatorname{IgG}$ ) in immunized mice. Transmissible gastroenteritis virus/porcine epidemic diarrhea virus (TGEV/PEDV)-specific sIgA antibody levels in the vagina $(\mathbf{a}, \mathbf{b})$ and the feces $(\mathbf{c}, \mathbf{d})$ post-immunization. Anti-TGEV IgG antibodies (e) and anti-PEDV-specific IgG antibodies (f) in immunized mice were detected at different time points. Bars represent the mean \pm standard error of each group. ${ }^{*} p<0.05,{ }^{* *} p<0.01$ vs. phosphate-buffered saline (PBS) and vector control groups; \# $p<0.05$, \#\# $p<0.01$ vs. rLpPG ${ }^{\mathrm{F}}-\mathrm{T7g} 10-\mathrm{eGFP}-6 \mathrm{D}-\mathrm{COE}$ group.

\subsection{TGEV and PEDV Neutralization}

The neutralizing capacities of the serum antibodies induced in mice orally immunized with recombinant strains against TGEV (Figure 6a) and PEDV (Figure 6b) showed inhibitory activity against viral infection. The anti-TGEV neutralizing antibody titers were $10^{-2}(1: 100)$ and $10^{-1.933}(1: 85.7)$ and the anti-PEDV neutralizing antibody titers were $10^{-1.967}$ (1:92.7) and $10^{-2.05}(1: 112.2)$ in mice immunized with rLpPG ${ }^{\mathrm{F}}-\mathrm{T7g} 10-\mathrm{eGFP}-6 \mathrm{D}-\mathrm{COE}$ and rLpPG-T7g10-6D-COE, respectively. These were significantly different from those obtained in the rLpPGF-T7g10 and PBS groups $(p<0.05)$. 

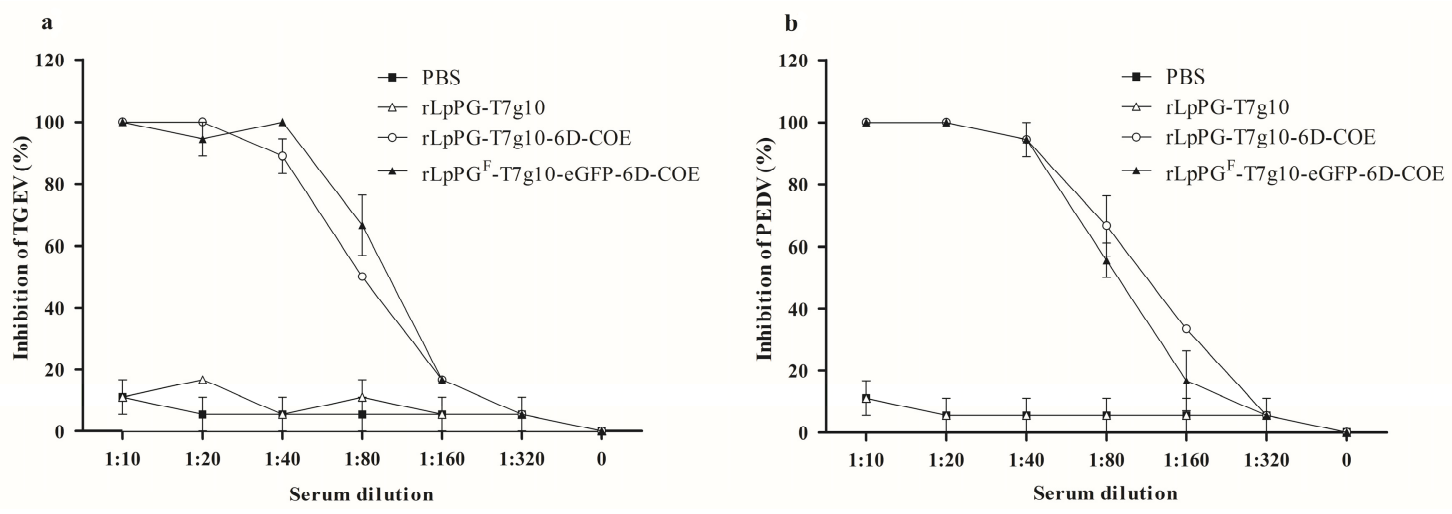

Figure 6. Neutralizing capacities of serum antibodies in immunized mice. Anti-PEDV (porcine epidemic diarrhea virus) (a), and anti-TGEV (transmissible gastroenteritis virus) (b) neutralizing antibodies in immunized mice were detected by plaque reduction assay with different serum dilutions from blood samples taken at 35 days post-immunization.

\subsection{Lymphocyte Proliferation}

The proliferation of spleen lymphocytes upon stimulation with purified COE or $6 \mathrm{D}$ proteins was analyzed by an MTT assay. The results showed that the proliferation levels of spleen lymphocytes from mice orally immunized with rLpPG-T7g10-6D-COE and rLpPG ${ }^{\mathrm{F}}$-T7g10-eGFP-6D-COE were significantly higher than those of spleen lymphocytes from mice in the rLpPGF-T7g10 and PBS control groups $(p<0.01)$. There was no significant difference $(p>0.05)$ observed between the rLpPG-T7g10-6D-COE and rLpPG ${ }^{\mathrm{F}}$-T7g10-eGFP-6D-COE groups (Figure 7).
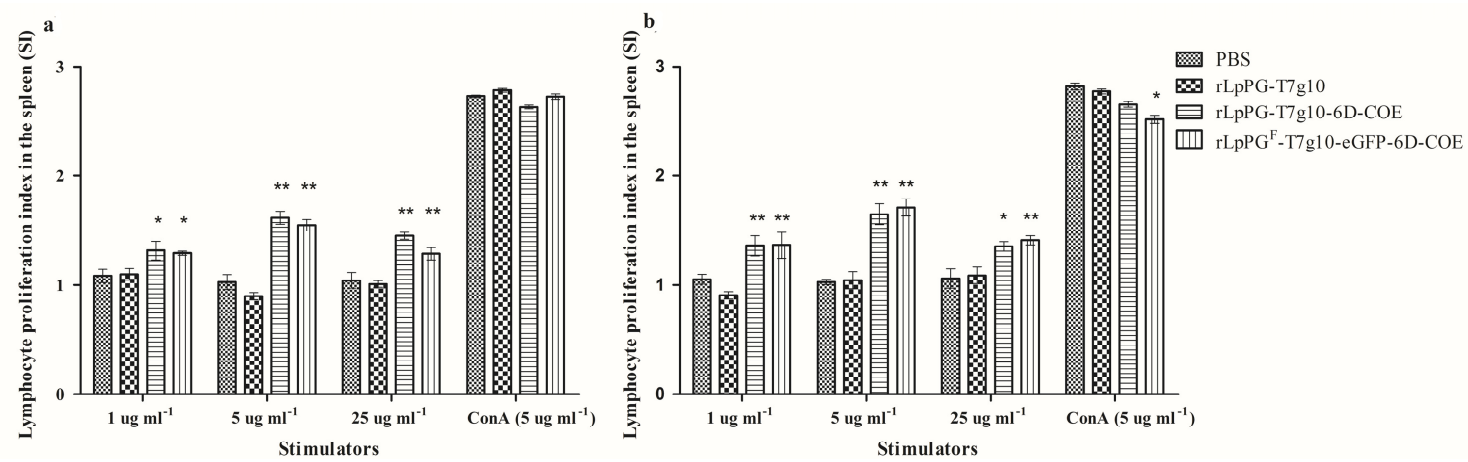

Figure 7. Proliferation of $\mathrm{T}$ lymphocytes from immunized mice. Lymphocyte proliferation was determined by 3-(4,5-dimethylthylthiazol-2-yl)-2,5-diphenyltetrazoliumbromide (MTT) assay with recombinant core neutralizing epitope (COE) protein $(\mathbf{a})$ or D protein $(\mathbf{b})$ as stimulating agents and concanavalin A (ConA) as a positive control. ${ }^{*} p<0.05,{ }^{* *} p<0.01$ vs. phosphate-buffered saline (PBS) and vector control groups.

\section{Discussion}

Over the past decades, large-scale outbreaks of diarrhea in swine caused by PEDV and TGEV have occurred in America, Europe, and Asia, resulting in considerable economic losses to the pig industry; in particular, PEDV infection has been responsible for many of these outbreaks [7,8]. The PEDV strain HLJ-2012 used in this study was isolated from an outbreak of acute diarrhea in piglets in Hegang, Heilongjiang Province, China [29], and phylogenetic analysis based on the $S$ gene revealed that HLJ-2012 shares high homology with U.S. PEDV strains. Both belong to the GIIa subgroup, which represents epidemic and pandemic field strains $[29,36]$. Occasionally, changes in the antigenicity of PEDV due to amino acid mutations have resulted in vaccination failure $[5,7]$. The $S$ protein neutralizing 
antigenic epitopes of PEDV have been reported to provide cross-protection against variant strains $[9,29]$. Therefore, we selected the COE of the PEDV S protein as an immunogen for developing an effective vaccine against $P E D V$ infection.

PEDV and TGEV infections initially occur on mucosal surfaces, especially the intestinal mucosal epithelial surface. Therefore, mucosal vaccination is an effective strategy for preventing viral diarrheal diseases [12]. In this study, we used L. casei to deliver the TGEV S protein protective $\mathrm{D}$ antigenic site (repeated six times) and the PEDV S protein COE for developing an oral mucosal vaccine against PEDV and TGEV. Our results suggested that the genetically engineered L. casei strain rLpPGF - T7g10-eGFP-6D-COE can be used as a bivalent oral vaccine for PEDV and TGEV, eliciting mucosal and humoral immune responses against both TGEV and PEDV via oral immunization. This was evidenced by significantly higher levels of virus-neutralizing antibodies, anti-PEDV/TGEV serum IgG, and mucosal sIgA in mice orally immunized with LpPGF $^{\mathrm{F}}$-T7g10-eGFP-6D-COE, compared to the levels for the rLpPG-T7g10 or PBS groups. Moreover, the genetically engineered L. casei vaccine is safe and easy to administer, making it practical and convenient.

Mucosal immunity plays an important role in preventing viral diarrheal diseases, and $\operatorname{sg} \mathrm{A}$ antibodies from durable lactogenic immunity are a good way for piglets to obtain passive immunoprotection, indicating the importance of the sIgA antibody in the control of viral infection. At the same time, the level of sIgA can reflect the status of viral infection and the protective efficacy of vaccines [34]. In the present study, anti-TGEV/PEDV sIgA antibodies could be effectively induced at high levels in the feces and vaginas of mice orally treated with rLpPGF-T7g10-eGFP-6D-COE. IgA is reported to peak at 6 weeks and decline at 8 weeks in piglets [26]; thus, if durable immunity was present at 35 days post-immunization, newborn pigs could obtain immune protection. Therefore, we assessed the levels of the sIgA antibody for 35 days post-immunization. Our data showed that the levels of sIgA in the feces of mice orally immunized with $\mathrm{rLPFG}^{\mathrm{F}}$-T7g10-eGFP-6D-COE gradually increased and peaked at 7, 21 and 35 days post-immunization, indicating that a mucosal immune response can be effectively elicited by rLpPG ${ }^{\mathrm{F}}-\mathrm{T7g} 10-\mathrm{eGFP}-6 \mathrm{D}-\mathrm{COE}$ after oral immunization. Therefore, the developed vaccine provides a promising strategy for protecting piglets from PEDV and TGEV infection via oral immunization.

Moreover, the neutralizing activity of an antibody is an important index used for evaluating the immunoprotective efficacy of a vaccine. In this study, high levels of serum antibody were elicited following oral immunization with rLpPG ${ }^{\mathrm{F}}$-T7g10-eGFP-6D-COE. This could effectively neutralize the PEDV/TGEV infection, and higher antibody titers reflected higher neutralizing activities. As a comparison, the neutralizing antibody titer induced by the recombinant $L$. casei oral vaccine developed in this study was higher than that induced by a previously developed DNA vaccine [24]. Therefore, oral immunization with genetically engineered L. casei strain rLPPG $^{\mathrm{F}}$-T7g10-eGFP-6D-COE may provide effective protection for piglets against PEDV and TGEV infection.

In addition, our results showed that the eGFP-marked recombinant Lactobacillus oral vaccine LPPG $^{\mathrm{F}}$-T7g10-eGFP-6D-COE (antibiotic-free selective marker vaccine) exhibited a similar immunogenicity to the antibiotic resistance marker vaccine rLpPG-T7g10-6D-COE, as the levels of antibodies induced by the rLpPG ${ }^{\mathrm{F}}$-T7g10-eGFP-6D-COE and rLpPG-T7g10-6D-COE vaccines were not significantly different $(p>0.05)$. Notably, the use of eGFP as a selective marker in rLpPG ${ }^{\mathrm{F}}$-T7g10-eGFP-6D-COE would avoid the main disadvantage of traditional plasmid expression systems by eliminating the use of antibiotic resistance genes as selective markers for genetically engineered bacteria $[18,24,25]$. Moreover, $\mathrm{rLpPG}^{\mathrm{F}}-\mathrm{T7g} 10-\mathrm{eGFP}-6 \mathrm{D}-\mathrm{COE}$ was constructed with a constitutive expression plasmid developed by our lab, exhibiting a significant advantage to inducible gene expression systems that require the use of an inductive agent. Additionally, a $p g s A$-derived anchoring matrix from Bacillus subtilis $[37,38]$ was used to express the fusion proteins, which were displayed on the bacterial surface, eliciting good immunogenicity. The improved plasmid expression system used in this study therefore provides a powerful tool for the development of recombinant Lactobacillus oral vaccines. 


\section{Conclusions}

In conclusion, an eGFP-marked recombinant Lactobacillus oral vaccine, rLpPG ${ }^{\mathrm{F}}$-T7g10-eGFP-6D-COE, was constructed in this study and provides a promising strategy for the development of a bivalent oral vaccine against TGEV and PEDV infection. Further investigations are underway to evaluate the immunogenicity of this vaccine following its oral administration in piglets and to optimize the immunization procedures for effective control of TGE and PED.

Acknowledgments: This work was supported by the National Key R and D Program of China (2017YFD0501105), the National Natural Sciences Funds (31472226), and the National Science and Technology Support Program in Rural Areas of the 12th Five-Year (2015BAD12B02-7).

Author Contributions: Yijing Li and Yigang $\mathrm{Xu}$ conceived and designed the study. Meiling Yu, Sunting Ma, Xiaona Wang, Yusai Wang, Ya Xiao, and Yanping Jiang performed the experiments. Li Wang, Xinyuan Qiao and Lijie Tang analyzed the data. Meiling Yu wrote the paper. All authors read and approved the manuscript.

Conflicts of Interest: The authors declare no conflict of interest.

\section{References}

1. Spaan, W.; Cavanagh, D.; Horzinek, M.C. Coronaviruses: Structure and genome expression. J. Gen. Virol. 1988, 69, 2939-2952. [CrossRef] [PubMed]

2. Doyle, L.P.; Hutchings, L.M. A transmissible gastroenteritis in pigs. J. Am. Vet. Med. Assoc. 1946, 108, 257-259. [PubMed]

3. Pensaert, M.; Callebaut, P.; Vergote, J. Isolation of a porcine respiratory, non-enteric coronavirus related to transmissible gastroenteritis. Vet. Q. 1986, 8, 257-261. [CrossRef] [PubMed]

4. Sasahara, J.; Harada, K.; Hayashi, S.; Watanabe, M. Studies on transmissible gastroenieritis in pig in Japan. Vet. Sci. 1958, 20, 1-6.

5. Lee, S.; Lee, C. Outbreak-related porcine epidemic diarrhea virus strains similar to US strains, South Korea, 2013. Emerg. Infect. Dis. 2014, 20, 1223-1226. [CrossRef] [PubMed]

6. Mole, B. Deadly pig virus slips through US borders. Nature 2013, 499, 388. [CrossRef] [PubMed]

7. Van Diep, N.; Norimine, J.; Sueyoshi, M.; Lan, N.T.; Hirai, T.; Yamaguchi, R. US-like isolates of porcine epidemic diarrhea virus from Japanese outbreaks between 2013 and 2014. Springerplus 2015, 4, 756. [CrossRef] [PubMed]

8. Boniotti, M.B.; Papetti, A.; Lavazza, A.; Alborali, G.; Sozzi, E.; Chiapponi, C.; Faccini, S.; Bonilauri, P.; Cordioli, P.; Marthaler, D. Porcine epidemic diarrhea virus and discovery of a recombinant swine enteric coronavirus, Italy. Emerg. Infect. Dis. 2016, 22, 83-87. [CrossRef] [PubMed]

9. Chen, J.; Liu, X.; Shi, D.; Shi, H.; Zhang, X.; Li, C.; Chi, Y.; Feng, L. Detection and molecular diversity of spike gene of porcine epidemic diarrhea virus in China. Viruses 2013, 5, 2601-2613. [CrossRef] [PubMed]

10. Chiou, H.Y.; Huang, Y.L.; Deng, M.C.; Chang, C.Y.; Jeng, C.R.; Tsai, P.S.; Yang, C.; Pang, V.F.; Chang, H.W. Phylogenetic analysis of the spike $(S)$ gene of the new variants of porcine epidemic diarrhoea virus in Taiwan. Transbound. Emerg. Dis. 2017, 64, 157-166. [CrossRef] [PubMed]

11. Vlasova, A.N.; Marthaler, D.; Wang, Q.H.; Culhane, M.R.; Rossow, K.D.; Rovira, A.; Collins, J.; Saif, L.J. Distinct characteristics and complex evolution of PEDV strains, North America, may 2013-February. Emerg. Infect. Dis. 2014, 20, 1620-1628. [CrossRef] [PubMed]

12. Jiang, X.P.; Hou, X.Y.; Tang, L.J.; Jiang, Y.P.; Ma, G.P.; Li, Y.J. A phase trial of the oral Lactobacillus casei vaccine polarizes Th2 cell immunity against transmissible gastroenteritis coronavirus infection. Appl. Microbiol. Biotechnol. 2016, 100, 7457-7469. [CrossRef] [PubMed]

13. Wang, X.L.; Wang, Z.B.; Xu, H.Y.; Xiang, B.; Dang, R.Y.; Yang, Z.Q. Orally administrated whole yeast vaccine against porcine epidemic diarrhea virus induced high levels of IgA response in mice and piglets. Viral Immunol. 2016, 29, 526-531. [CrossRef] [PubMed]

14. Bohl, E.H.; Gupta, R.K.; Olquin, M.V.; Saif, L.J. Antibody responses in serum, colostrum, and milk of swine after infection or vaccination with transmissible gastroenteritis virus. Infect. Immun. 1972, 6, 289-301. [PubMed] 
15. Mutwiri, G.; Watts, T.; Lew, L.; Beskorwayne, T.; Papp, Z.; Bace-estrada, M.E.; Griebel, P. Ileal and jejunal Peyer's patches play distinct roles in mucosal immunity of sheep. Immunology 1999, 97, 455-461. [CrossRef] [PubMed]

16. Song, D.S.; Oh, J.S.; Kang, B.K.; Yang, J.S.; Moon, H.J.; Yoo, H.S.; Jang, Y.S.; Park, B.K. Oral efficacy of Vero cell attenuated porcine epidemic diarrhea virus DR13 strain. Res. Vet. Sci. 2007, 82, 134-140. [CrossRef] [PubMed]

17. Song, D.; Moon, H.; Kang, B. Porcine epidemic diarrhea: A review of current epidemiology and available vaccines. Clin. Exp. Vaccine Res. 2015, 4, 166-176. [CrossRef] [PubMed]

18. Ge, J.W.; Liu, D.Q.; Li, Y.J. Construction of recombinant lactobacilli expressing the core neutralizing epitope (COE) of porcine epidemic diarrhea virus and a fusion protein consisting of COE and Escherichia coli heat-labile enterotoxin $\mathrm{B}$, and comparison of the immune responses by orogastric immunization. Can. J. Microbiol. 2012, 58, 1258-1267. [PubMed]

19. Posthumus, W.P.; Lenstra, J.A.; Schaaper, W.M.; van Nieuwstadt, A.P.; Enjuanes, L.; Meloen, R.H. Analysis and simulation of a neutralizing epitope of transmissible gastroenteritis virus. J. Virol. 1990, 64, 3304-3309. [PubMed]

20. Seegers, J.F. Lactobacilli as live vaccine delivery vectors: Progress and prospects. Trends Biotechnol. 2002, 20, 508-515. [CrossRef]

21. Yu, M.L.; Qi, R.M.; Chen, C.Y.; Yin, J.Y.; Ma, S.T.; Shi, W.; Wu, Y.; Ge, J.W.; Jiang, Y.P.; Tang, L.J.; et al. Immunogenicity of recombinant Lactobacillus casei expressing F4 (K88) fimbrial adhesin FaeG in conjunction with a heat-labile enterotoxin A (LTAK63) and heat-labile enterotoxin B (LTB) of enterotoxigenic Escherichia coli as an oral adjuvant in mice. J. Appl. Microbiol. 2017, 122, 506-515. [CrossRef] [PubMed]

22. Zhang, N.; Hou, X.; Yu, L.; Wang, G.; Zhao, Z.; Gao, Y. Colonization and distribution of recombinant Lactobacillus casei with green fluorescent protein in mice intestine. Wei Sheng Wu Xue Bao 2010, 50, 1232-1238. [PubMed]

23. Xu, Y.G.; Cui, L.C.; Tian, C.Y.; Zhang, G.C.; Huo, G.C.; Tang, L.J.; Li, Y.J. Immunogenicity of recombinant classic swine fever virus CD8 (+) T lymphocyte epitope and porcine parvovirus VP2 antigen coexpressed by Lactobacillus casei in swine via oral vaccination. Clin. Vaccine Immunol. 2011, 18, 1979-1986. [CrossRef] [PubMed]

24. Meng, F.D.; Ren, Y.D.; Suo, S.; Sun, X.J.; Li, X.L.; Li, P.C.; Yang, W.; Li, G.X.; Li, L.; Schwegmann-Wessels, C.; et al. Evaluation on the efficacy and immunogenicity of recombinant DNA plasmids expressing spike genes from porcine transmissible gastroenteritis virus and porcine epidemic diarrhea virus. PLoS ONE 2013, 8 , e57468. [CrossRef] [PubMed]

25. Walsh, C.; Walsh, C. Antibiotics: Actions, origins, resistance. Nat. Prod. Rep. 2005, 22, 304-305.

26. Zhang, Y.; Zhang, X.; Liao, X.; Huang, X.; Cao, S.; Wen, X.; Wen, Y.; Wu, R.; Liu, W. Construction of a bivalent DNA vaccine co-expressing $S$ genes of transmissible gastroenteritis virus and porcine epidemic diarrhea virus delivered by attenuated Salmonella typhimurium. Virus Genes 2016, 52, 354-364. [CrossRef] [PubMed]

27. Elliott, D.A.; Braam, S.R.; Koutsis, K.; Ng, E.S.; Jenny, R.; Lagerqvist, E.L.; Biben, C.; Hatzistavrou, T.; Hirst, C.E.; Yu, Q.C.; et al. NKX2-5(eGFP/w) hESCs for isolation of human cardiac progenitors and cardiomyocytes. Nat. Methods 2011, 8, 1037-1040. [CrossRef] [PubMed]

28. Kemter, E.; Cohrs, C.M.; Schäfer, M.; Schuster, M.; Steinmeyer, K.; Wolf-van, B.L.; Wolf, A.; Wuensch, A.; Kurome, M.; Kessler, B.; et al. INS-eGFP transgenic pigs: A novel reporter system for studying maturation, growth and vascularisation of neonatal islet-like cell clusters. Diabetologia 2017, 60, 1152-1156. [CrossRef] [PubMed]

29. Li, Y.J.; Zhang, B.; Tang, L.J.; Jiang, Y.P.; Chen, P.P. Genetic variation analysis of $S 1$ genes of PEDV endemic strains and immunogenicity detection of HLJ-2012 strain. J. Northeast Agric. Univ. 2014, 45, 1-9.

30. Ren, X.F.; Li, Y.J.; Liu, B.Q. Cloning and homology comparison of $S$ gene for isolate TH-98 of porcine transmissible gastroenteritis virus. Agric. Sci. China 2002, 2, 314-320.

31. Liu, D.Q.; Qiao, X.Y.; Ge, J.W.; Tang, L.J.; Jiang, Y.P.; Li, Y.J. Construction and characterization of Lactobacillus pentosus expressing the $\mathrm{D}$ antigenic site of the spike protein of transmissible gastroenteritis virus. Can. J. Microbiol. 2011, 57, 392-397.

32. Wang, C.; Lian, G.H.; Zhao, L.L.; Wu, Y.; Li, Y.J.; Tang, L.J.; Qiao, X.Y.; Jiang, Y.P.; Liu, M. Virulence and serological studies of recombinant infectious hematopoietic necrosis virus (IHNV) in rainbow trout. Virus Res. 2016, 220, 193-202. [CrossRef] [PubMed] 
33. Song, B.F.; Ju, L.Z.; Li, Y.J.; Tang, L.J. Chromosomal insertions in the Lactobacillus casei upp gene that are useful for vaccine expression. Appl. Environ. Microbiol. 2014, 80, 3321-3326. [CrossRef] [PubMed]

34. Jiang, X.P.; Yu, M.L.; Qiao, X.Y.; Liu, M.; Tang, L.J.; Jiang, Y.P.; Cui, W.; Li, Y.J. Up-regulation of MDP and tuftsin gene expression in Th1 and Th17 cells as an adjuvant for an oral Lactobacillus casei vaccine against anti-transmissible gastroenteritis virus. Appl. Microbiol. Biotechnol. 2014, 98, 8301-8312. [CrossRef] [PubMed]

35. Robinson, K.; Chamberlain, L.M.; Schofield, K.M.; Wells, J.M.; Le Page, R.W. Oral vaccination of mice against tetanus with recombinant Lactococcus lactis. Nat. Biotechnol. 1997, 15, 653-657. [CrossRef] [PubMed]

36. Shi, W.; Jia, S.; Zhao, H.Y.; Yin, J.Y.; Wang, X.N.; Yu, M.L.; Ma, S.T.; Wu, Y.; Chen, Y.; Fan, W.L.; et al. Novel approach for isolation and identification of porcine epidemic diarrhea virus (PEDV) strain NJ using porcine intestinal epithelial cells. Viruses 2017, 9, 19. [CrossRef] [PubMed]

37. Ashiuchi, M.; Nawa, C.; Kamei, T.; Song, J.J.; Hong, S.P.; Sung, M.H.; Soda, K.; Misono, H. Physiological and biochemical characteristics of poly gamma-glutamate synthetase complex of Bacillus subtilis. Eur. J. Biochem. 2001, 268, 5321-5328. [CrossRef] [PubMed]

38. Van, B.J.; Blohmke, C.J.; Folkerts, H.; de Boer, R.; Zakrzewska, A.; Kulik, W.; Vaz, F.M.; Brul, S.; Ter Beek, A. RodZ and PgsA play intertwined roles in membrane homeostasis of Bacillus subtilis and resistance to weak organic acid stress. Front. Microbiol. 2016, 7, 1633. [CrossRef]

(C) 2017 by the authors. Licensee MDPI, Basel, Switzerland. This article is an open access article distributed under the terms and conditions of the Creative Commons Attribution (CC BY) license (http:/ / creativecommons.org/licenses/by/4.0/). 